\title{
Infinitely stiff composite via a rotation-stabilized negative-stiffness phase
}

\author{
D. M. Kochmann ${ }^{1, a)}$ and W. J. Drugan ${ }^{2, b)}$ \\ ${ }^{1}$ Graduate Aerospace Laboratories, California Institute of Technology, Pasadena, CA 91125, USA \\ ${ }^{2}$ Engineering Physics, University of Wisconsin-Madison, 1500 Engineering Drive, Madison, WI 53706, USA
}

(Received 13 May 2011; accepted 20 June 2011; published online 7 July 2011)

\begin{abstract}
We show that an elastic composite material having a component with sufficiently negative stiffness to produce positive-infinite composite stiffness can be stabilized by the gyroscopic forces produced by composite rotation. (C) 2011 American Institute of Physics. [doi:10.1063/1.3609328]
\end{abstract}

Combining several materials ("phases") into a composite material permits creation of new materials with tunable overall response, e.g., elastic properties, which can be related to the elastic moduli, arrangement, and concentrations of the composite's phases. ${ }^{1}$ A recent advance ${ }^{2}$ predicts use of one phase having correctly tuned non-positive-definite elastic moduli (i.e., negative stiffness) in a two-phase composite can produce theoretically infinite composite stiffness. Employing this theory, the Lakes group ${ }^{3}$ recently made such a composite (inclusion negative-stiffness produced by a constrained phase transformation), which exhibited momentary (unstable) extreme composite stiffness ten times that of diamond.

Although a homogeneous, free-standing body of negative stiffness is unstable by itself, ${ }^{4}$ we have recently shown ${ }^{5,6}$ that a negative-stiffness phase can be stabilized if it is an inclusion completely encapsulated by a sufficiently stiff matrix material. However, the range of negative inclusion stiffness so stabilized is not sufficient to allow for the tuning required to produce stable theoretically infinite overall composite stiffness. We therefore seek strategies to enlarge the stability regime of composite materials containing a negative-stiffness phase.

Here we illustrate one such strategy: use of dynamic excitation. It is known that appropriate dynamic excitation can broaden a system's stability regime; a famous example is the inverted pendulum. ${ }^{7}$ (Inertia can also do this. ${ }^{8}$ ) We analyze here the instructive case of a rotating composite (whose nonrotating dynamic stability regime is $k_{n o w n}{ }^{6}$ ). We employ a full dynamic analysis to derive the restrictions on the elastic moduli of the phases in the rotating composite that guarantee composite stability. We show that for sufficient rates of rotation, the composite will remain stable even when the inclusion stiffness is sufficiently negative to produce theoretically positive-infinite composite stiffness.

The composite to be analyzed is comprised of two homogeneous, isotropic, linear elastic phases: an infinitely long, circular cylinder (radius $a$, Lamé moduli $\lambda_{1}, \mu_{1}$ ), embedded in a concentric coating (outer radius $b$, Lamé moduli $\left.\lambda_{2}, \mu_{2}\right)$. The composite is in a state of plane strain with deformations confined to the $x, y$-plane, and it rotates with angular velocity $\Omega$ about the $z$-axis with the center of rotation at the origin (see Fig. 1). For simplicity and clarity, we have chosen the simplest realistic solid composite geometry to illus-

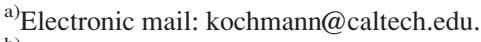

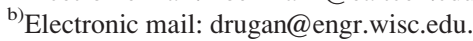

trate the phenomenon described herein; clearly, this phenomenon can be made to occur in more geometrically complex composite materials when subjected to appropriate dynamic excitation.

The dynamic governing equations for a homogeneous, isotropic, linear elastic solid with Lamé moduli $\lambda, \mu$, when no body forces act, are the Navier equations

$$
(\lambda+\mu) \nabla(\nabla \cdot \boldsymbol{u})+\mu \nabla^{2} \boldsymbol{u}=\rho \boldsymbol{a}
$$

where $\boldsymbol{u}(\boldsymbol{x}, t)$ is the infinitesimal displacement field (depending on position $x$ and time $t$ ) relative to the rotating reference frame, $\rho$ is mass density, and $\boldsymbol{a}(\boldsymbol{x}, t)$ is the total acceleration, which for a rotating solid decomposes as

$$
\boldsymbol{a}=\ddot{\boldsymbol{u}}+\Omega \times[\Omega \times(\boldsymbol{x}+\boldsymbol{u})]+\dot{\boldsymbol{\Omega}} \times(\boldsymbol{x}+\boldsymbol{u})+2 \Omega \times \dot{\boldsymbol{u}},
$$

with a dot denoting material time derivative. The first term is relative acceleration in the rotating system, the second and third terms are acceleration due to rigid body rotation ( $x$ is undeformed position), and the final term is Coriolis acceleration. Terms explicitly involving $\boldsymbol{x}$ only give rise to a particular solution of the partial differential equations, leaving overall stability unaffected; we thus omit them in the stability analysis, keeping only those terms characterizing free vibration about the rotating steady-state. For simplicity, we assume a constant $\Omega$.

Using polar coordinates $r$ and $\varphi$ for convenience (see Fig. 1), the Navier equations become

$$
\begin{gathered}
c_{p}^{2}\left(u_{r, r r}+\frac{u_{r, r}}{r}-\frac{u_{r}}{r^{2}}\right)=\ddot{u}_{r}-2 \Omega \dot{u}_{\varphi}-\Omega^{2} u_{r}, \\
c_{s}^{2}\left(u_{\varphi, r r}+\frac{u_{\varphi, r}}{r}-\frac{u_{\varphi}}{r^{2}}\right)=\ddot{u}_{\varphi}+2 \Omega \dot{u}_{r}-\Omega^{2} u_{\varphi},
\end{gathered}
$$

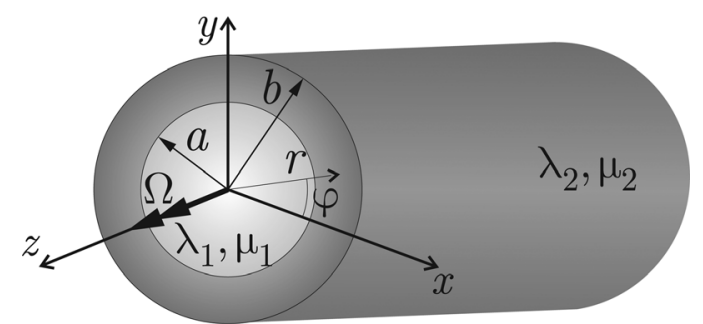

FIG. 1. The rotating coated-cylinder composite. 
where a subscript comma denotes partial differentiation with respect to the ensuing variable(s), and $c_{p}=\sqrt{(\lambda+2 \mu) / \rho}$, $c_{s}=\sqrt{\mu / \rho}$ are the elastic wave speeds. We introduce the abbreviations $\beta=\mu /(\lambda+2 \mu), \Gamma=\Omega / \omega$.

The general solution representation of the displacement field in polar coordinates for a homogeneous, rotating body (that is, the general solution of the above pair of Navier equations) is given by

$$
\boldsymbol{u}(r, \varphi, t)=\sum_{m=-\infty}^{\infty} \boldsymbol{v}(r ; m) e^{i m \varphi} e^{i \omega_{m} t}
$$

with the following definitions:

$$
\begin{gathered}
v_{r}(r ; m)=C_{1}(m) f_{m}^{+}\left(\beta \Lambda_{+}, k_{+}\right)-C_{2}(m) i f_{m}^{-}\left(-\Lambda_{+}, k_{-}\right), \\
v_{\varphi}(r ; m)=C_{1}(m) i f_{m}^{-}\left(\beta \Lambda_{+}, k_{+}\right)+C_{2}(m) f_{m}^{+}\left(-\Lambda_{+}, k_{-}\right), \\
f_{m}^{ \pm}(\alpha, k)=(1+\alpha) J_{m-1}(k r) \pm(1-\alpha) J_{m+1}(k r) \\
k_{ \pm}^{2}=\left[(1+\beta)\left(1+\Gamma^{2}\right) \pm \Theta\right] \omega_{m}^{2} / 2 c_{s}^{2}, \\
\Lambda_{ \pm}=4 \Gamma /\left[(1-\beta)\left(1+\Gamma^{2}\right) \pm \Theta\right] \\
\Theta=\sqrt{(1+\beta)^{2}\left(1+\Gamma^{2}\right)^{2}-4 \beta\left(1-\Gamma^{2}\right)^{2}}
\end{gathered}
$$

if the material satisfies plane strain positive-definiteness ${ }^{9}$ (i.e., if the elastic moduli satisfy $\mu>0$ and $\lambda>-\mu$ ). The solution must be modified by switching signs in $k_{ \pm}$and $\Lambda_{ \pm}$if the material violates positive-definiteness while remaining at least strongly elliptic (i.e., if $\mu>0$ and $-2 \mu<\lambda<-\mu$ ). $J_{m}(x)$ is the order- $m$ Bessel function of the first kind, $i=\sqrt{-1}$, and $C_{j}(m)$ are complex, constant coefficients (one set for each mode $m$ ).

For the rotating composite, independent displacement field representations are necessary in the inclusion and coating: the inclusion displacements $\boldsymbol{u}_{\text {inc }}$ take the above form with the inclusion's material properties and the signs switched in $k_{ \pm}$and $\Lambda_{ \pm}$, while the displacements $\boldsymbol{u}_{\text {mat }}$ in the coating (matrix) material have the above form with the coating's material properties and extended by the addition of analogous Bessel-Y solutions, with different coefficients $C_{j}(m), j=3, \ldots, 6$. Both materials have the same mass density. Changing the mass density of either material does not alter the stability limit. ${ }^{6}$

The needed stress components are obtained via Hooke's law from the displacement field representation for each material independently $\left(\boldsymbol{\sigma}_{\text {inc }}\right.$ and $\left.\boldsymbol{\sigma}_{\text {mat }}\right)$ as

$$
\begin{aligned}
& \sigma_{r r}=(\lambda+2 \mu) u_{r, r}+\lambda\left(u_{r}+u_{\varphi, \varphi}\right) / r, \\
& \sigma_{r \varphi}=\mu\left[u_{\varphi, r}+\left(u_{r, \varphi}-u_{\varphi}\right) / r\right] .
\end{aligned}
$$

This results in stresses in both materials of the form

$$
\boldsymbol{\sigma}(r, \varphi, t)=\sum_{m=-\infty}^{\infty} \boldsymbol{\Sigma}(r ; m) e^{i m \varphi} e^{i \omega_{m} t}
$$

Stability analysis requires determining the rotating composite cylinder's free vibration eigenfrequencies. We apply zero tractions on the outer surface of the coating, and traction and displacement continuity across the inclusion/coating interface. These conditions hold for all $\varphi$, so they can be imposed independently for each $m$-mode

$$
\begin{array}{r}
\boldsymbol{\Sigma}_{\text {mat }}(b ; m) \cdot \boldsymbol{n}=\mathbf{0}, \\
{\left[\boldsymbol{\Sigma}_{\text {mat }}(a ; m)-\boldsymbol{\Sigma}_{\text {inc }}(a ; m)\right] \cdot \boldsymbol{n}=\mathbf{0},} \\
\boldsymbol{v}_{\text {mat }}(a ; m)=\boldsymbol{v}_{\text {inc }}(a ; m),
\end{array}
$$

where $\boldsymbol{n}$ is the radial unit vector. Writing these six component equations (to be solved for the six unknown coefficients $\left.C_{j}(m)\right)$ in the matrix form $\boldsymbol{M}(\omega ; m) \cdot \boldsymbol{C}(m)=\mathbf{0}$, we obtain for each mode $m$ the infinite set $\Psi_{m}$ of eigenfrequencies from the non-trivial solution, i.e.,

$$
\Psi_{m}=\{\omega \mid \operatorname{det}[\boldsymbol{M}(\omega ; m)]=0\} .
$$

The characteristic equation $\operatorname{det}[\boldsymbol{M}(\omega ; m)]=0$ is solved numerically for the eigenfrequencies of a specific mode $m$, with given elastic moduli $\lambda_{i}$ and $\mu_{i}$, and radii $a$ and $b$. (Results depend only on ratios of the elastic moduli and the radius ratio $a / b$, not on their individual values.) Dynamic stability of the composite requires all eigenfrequencies to have positive imaginary parts (to avoid displacements that grow with time, indicating instability). Thus, we numerically check for any given combination of composite parameters whether unstable solutions $\operatorname{Im}(\omega)<0$ exist. Iterating this procedure for all moduli and radii combinations, we obtain a complete stability map.

The plane strain effective bulk modulus of the rotating composite, $\bar{\kappa}$, is (where $\overline{\boldsymbol{\sigma}}, \overline{\boldsymbol{\varepsilon}}$ are average stress and strain)

$$
\begin{aligned}
\bar{\kappa}= & \operatorname{tr} \overline{\boldsymbol{\sigma}} /(2 \operatorname{tr} \overline{\boldsymbol{\varepsilon}})=\left[\left(\lambda_{2}+\mu_{2}\right)\left(\lambda_{1}+\mu_{1}+\mu_{2}\right)\right. \\
& \left.+\left(\frac{a}{b}\right)^{2}\left(\lambda_{1}-\lambda_{2}+\mu_{1}-\mu_{2}\right) \mu_{2}\right] / \\
& {\left[\lambda_{1}+\mu_{1}+\mu_{2}+\left(\frac{a}{b}\right)^{2}\left(\lambda_{2}-\lambda_{1}+\mu_{2}-\mu_{1}\right)\right] . }
\end{aligned}
$$

Fig. 2 illustrates the (normalized) composite bulk modulus $\bar{\kappa} / \mu_{2}$ as a function of $\kappa_{1} / \mu_{1}$ and $\kappa_{2} / \mu_{2}$, showing that for an appropriately tuned negative inclusion bulk modulus, the

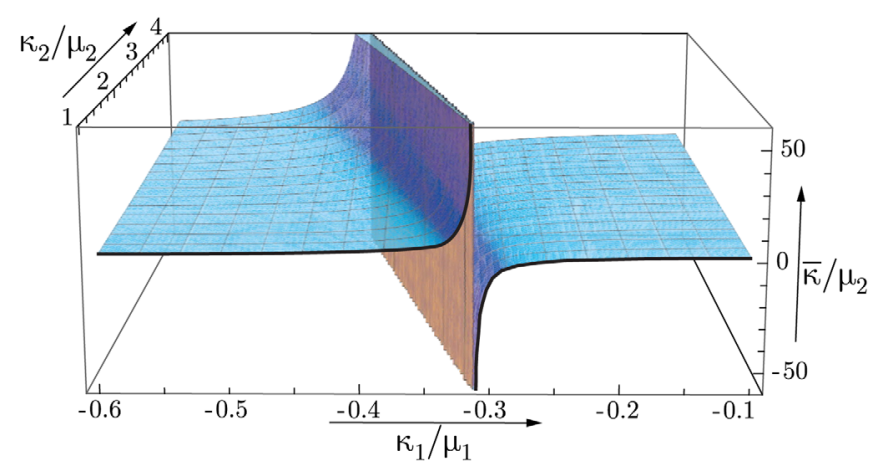

FIG. 2. (Color online) Normalized overall bulk modulus of the coated cylin$\operatorname{der} \bar{\kappa}$, for the example choices $\mu_{1} / \mu_{2}=4$ and $a / b=1 / 3$, partially illustrating the fact that theoretically positive-infinite composite bulk modulus can always be achieved by appropriate tuning of the (negative) inclusion bulk modulus $\kappa_{1}$. 


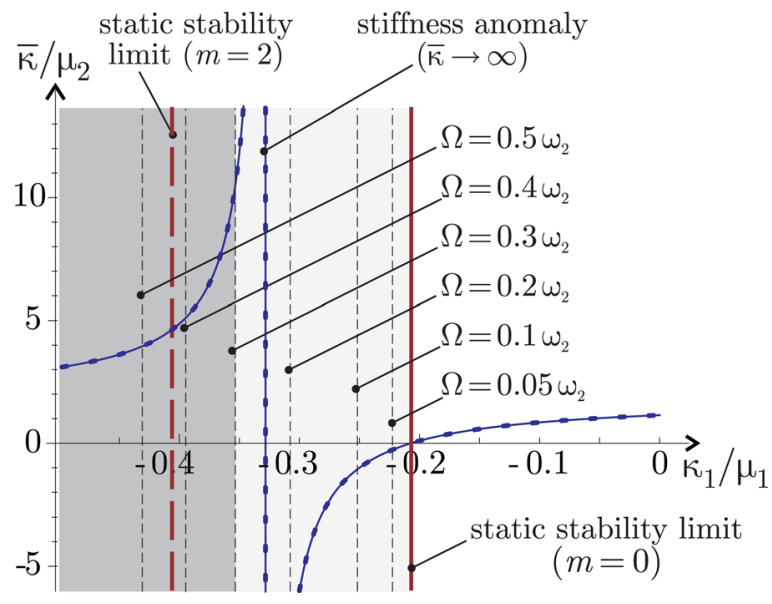

FIG. 3. (Color online) Normalized composite $\bar{\kappa}$ vs. inclusion $\kappa_{1}$ bulk moduli, and composite stability limits, for a composite with $\kappa_{2} / \mu_{2}=1.5$, $\mu_{1} / \mu_{2}=4, a / b=1 / 3$. The (light dashed) stability limits correspond to nonzero $\Omega$ values; the thick solid and dashed lines show the nonrotating (static) stability limits for modes $m=0$ and 2 , respectively. The white region is statically stable. The lightly shaded area shows the stability region expansion produced by rotating the composite at the angular frequency $\Omega=0.3 \omega_{2}$; note this expanded region comfortably includes the negative inclusion bulk modulus that produces theoretically positive-infinite composite bulk modulus.

overall (composite) bulk modulus becomes positive-infinite; stability must be examined separately.

The dynamic stability analysis of the nonrotating composite cylinder ${ }^{6}$ showed that each mode $m$ provides a unique stability limit on the elastic moduli for each radius ratio $a / b$. For a positive-definite coating encapsulating a negative-stiffness inclusion, the rotational-symmetric $m=0$ mode provides the strongest restriction on the elastic moduli and is therefore critical. The present analysis shows that the rotation of the composite stabilizes the critical $m=0$ mode to some extent but does not affect the higher modes. For geometric reasons (rigid body displacement must be excluded), the $m=1$ mode is discarded. Thus, the overall stable regime for the elastic moduli can be expanded by rotating the composite, with an upper limit arising from the $m=2$ mode.

Fig. 3 shows a slice through Fig. 2 at the example value $\kappa_{2} / \mu_{2}=1.5$, overlaid with the stability limits for various rotation frequencies $\Omega$. Positive-infinite composite bulk modulus occurs at $\kappa_{1} / \mu_{1}=-0.33$, which violates the stability limit of the nonrotating (static) composite of $\kappa_{1} / \mu_{1}>-0.21$. However, observe that increasing the rotation frequency of the composite shifts the composite stability limit to the left; when this frequency reaches $\Omega / \omega_{2}=0.24$, the composite stability regime is sufficiently expanded to permit positive-infinite composite bulk modulus while retaining composite stability. $\left(\omega_{2}=\sqrt{\mu_{2} / \rho} / b\right.$ is the natural frequency of the coating material.)

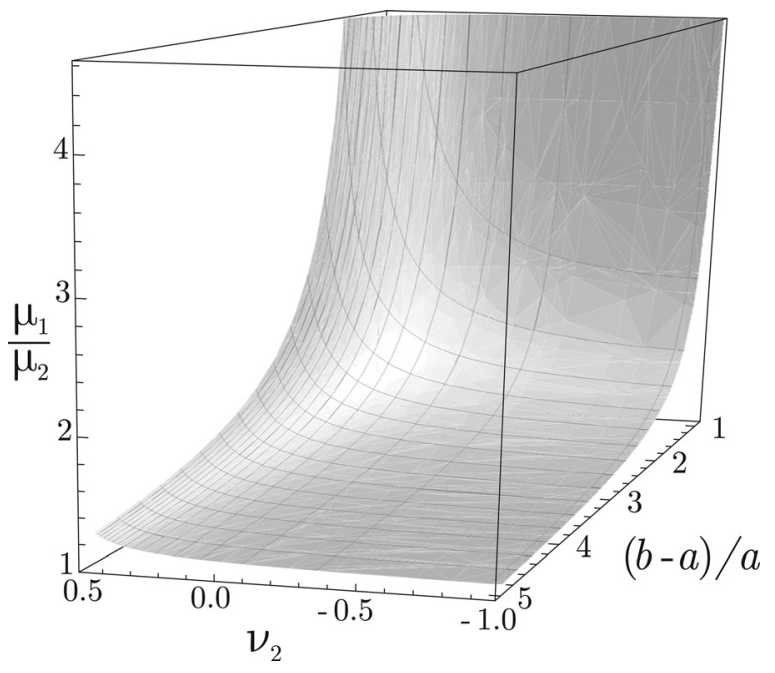

FIG. 4. Combinations of $\mu_{1} / \mu_{2},(b-a) / a$, and $v_{2}$, the coating's Poisson ratio, which permit stabilization via rotation of theoretically positive-infinite composite stiffness (stable combinations lie above the displayed surface).

We have determined in general the combinations of shear moduli and radii ratios for which stabilization of the theoretically positive-infinite composite stiffness response is possible via composite rotation. Fig. 4 shows the results: the surface separates stable combinations (lying above) from unstable ones. Clearly, theoretically positive-infinite composite stiffness can be stabilized by rotation of the composite for a wide range of coating thicknesses, shear modulus ratios, and coating Poisson's ratios. The results show that the inclusion's shear modulus must exceed that of the matrix material, and the coating's thickness must be at least as large as the inclusion radius; composites easiest to stabilize have high inclusion shear modulus, low matrix shear modulus, high matrix density, and dilute inclusion volume fraction.

We acknowledge support from NSF Grant DMR0949254 and ARO/DARPA Grant 57492-EG-DRP.

${ }^{1} \mathrm{G}$. W. Milton, Theory of Composites (Cambridge University Press, Cambridge, England, 2001).

${ }^{2}$ R. S. Lakes and W. J. Drugan, J. Mech. Phys. Solids 50, 979 (2002).

${ }^{3}$ T. Jaglinski, D. Kochmann, D. Stone, and R. S. Lakes, Science 315, 620 (2007).

${ }^{4}$ R. Hill, J. Mech. Phys. Solids 5, 229 (1957)

${ }^{5}$ W. J. Drugan, Phys. Rev. Let. 98, 055502 (2007).

${ }^{6}$ D. M. Kochmann and W. J. Drugan, J. Mech. Phys. Solids 57, 1122 (2009).

${ }^{7}$ J. A. Blackburn, H. J. T. Smith, and N. Gronbech-Jensen, Am. J. Phys. 60, 903 (1992).

${ }^{8}$ Y. C. Wang, J. G. Swadener, and R. S. Lakes, Philos. Mag. Lett. 86, 99 (2006).

${ }^{9}$ D. Censor and M. Schoenberg, Appl. Sci. Res. 27, 401 (1973). 